\title{
BIRMINGHAM
}

\section{Thrifty swimming with shear-thinning: a note on out-of-plane effects for undulatory locomotion through shear-thinning fluids}

Gagnon, David; Montenegro-Johnson, Thomas

DOI:

10.1017/S1446181118000032

License:

None: All rights reserved

Document Version

Peer reviewed version

Citation for published version (Harvard):

Gagnon, D \& Montenegro-Johnson, T 2018, 'Thrifty swimming with shear-thinning: a note on out-of-plane effects for undulatory locomotion through shear-thinning fluids', The ANZIAM Journal.

https://doi.org/10.1017/S1446181118000032

Link to publication on Research at Birmingham portal

\section{Publisher Rights Statement:}

(C) 2018 Australian Mathematical Society

Final Version of Record available at:

https://doi.org/10.1017/S1446181118000032

\section{General rights}

Unless a licence is specified above, all rights (including copyright and moral rights) in this document are retained by the authors and/or the copyright holders. The express permission of the copyright holder must be obtained for any use of this material other than for purposes permitted by law.

-Users may freely distribute the URL that is used to identify this publication.

- Users may download and/or print one copy of the publication from the University of Birmingham research portal for the purpose of private study or non-commercial research.

- User may use extracts from the document in line with the concept of 'fair dealing' under the Copyright, Designs and Patents Act 1988 (?)

- Users may not further distribute the material nor use it for the purposes of commercial gain.

Where a licence is displayed above, please note the terms and conditions of the licence govern your use of this document.

When citing, please reference the published version.

Take down policy

While the University of Birmingham exercises care and attention in making items available there are rare occasions when an item has been uploaded in error or has been deemed to be commercially or otherwise sensitive.

If you believe that this is the case for this document, please contact UBIRA@lists.bham.ac.uk providing details and we will remove access to the work immediately and investigate. 


\title{
Thrifty swimming with shear-thinning: a note on out-of-plane effects for undulatory locomotion through shear-thinning fluids
}

\author{
D. A. GAGNON ${ }^{\square} 1$ and T. D. MONTENEGRO-JOHNSON ${ }^{2}$
}

(Received xx Month 2017)

\begin{abstract}
Microscale propulsion is integral to numerous biomedical systems, for example biofilm formation and human reproduction, where the surrounding fluids comprise suspensions of polymers. These polymers endow the fluid with non-Newtonian rheological properties, such as shear-thinning and viscoelasticity. Thus, the complex dynamics of non-Newtonian fluids presents numerous modelling challenges. Here, we demonstrate that neglecting "out-of-plane" effects during swimming through a shear-thinning fluid results in a significant overestimate of fluid viscosity around the undulatory swimmer C. elegans. This miscalculation of viscosity corresponds with an overestimate of the power the swimmer expends, a key biophysical quantity important for understanding the internal mechanics of the swimmer. As experimental flow tracking techniques improve, accurate experimental estimates of power consumption in similar undulatory systems, such as the planar beating of human sperm through cervical mucus, will be required to probe the interaction between internal power generation, fluid rheology, and the resulting waveform.
\end{abstract}

2010 Mathematics subject classification: 76Z99.

Keywords and phrases: Low-Reynolds number swimming, Undulatory propulsion, $C$. elegans, Non-Newtonian Fluids.

\section{Introduction}

Despite a history of mathematical study dating back nearly 70 years to the work of G. I. Taylor [30], microscale propulsion remains an increasingly active topic in applied mathematics. The diversity and importance of life at the microscopic scale cannot be overestimated; there are over 1400 known human pathogens alone [1], accounting for much less than $1 \%$ of the total number of microbial species. In many cases, propulsion through fluids forms a key part of a microbe's life cycle, and in a biomedical

\footnotetext{
${ }^{1}$ Mechanical Engineering and Applied Mechanics, University of Pennsylvania, Philadelphia, USA; e-mail: david.gagnon@ georgetown.edu orcid:0000-0003-3122-080X.

${ }^{2}$ School of Mathematics, University of Birmingham, UK; e-mail: t.d.johnson@bham.ac.ukorcid:00000002-9370-7720.
}

(C) Australian Mathematical Society 2017, Serial-fee code 0334-2700/17 

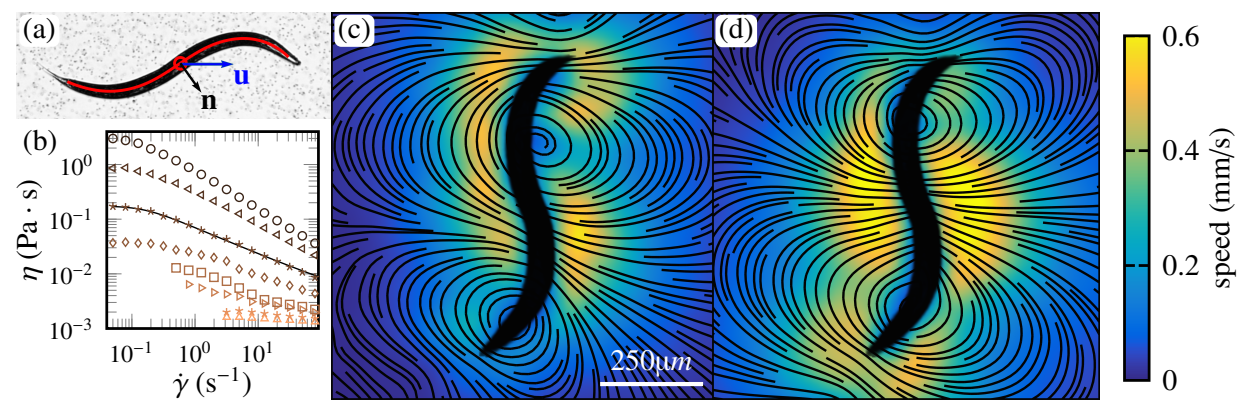

Figure 1. (Colour available online) a) The $1 \mathrm{~mm}$ long nematode $C$. elegans, with centreline and centroid in red and sample outward normal vector $\boldsymbol{n}$. b) Steady rheology curves showing viscosity $\eta$ versus shear rate $\dot{\gamma}$ for shear-thinning solutions of xanthan gum in buffer; increasing viscosity indicates increasing concentration, which ranges from 50-3000 ppm by weight. c) and d) A snapshot of the streamlines around a freely-swimming nematode in a Newtonian and shear-thinning fluid respectively. Colour represents fluid speed.

context can be central to disease virulence and mammalian fertility. Motility in Trypanosoma brucei, the parasite responsible for sleeping sickness, is integral to its development [12]. In Helicobacter pylori, motility improves initial colonization and leads to a more robust infection of gastritis and ulcers [21]. In contrast to the helical propulsion of many other bacteria, Borrelia burgdorferi, the spirochete responsible for Lyme disease, produces planar undulations allowing it to propel through the viscoelastic gel environment of the mammalian dermis [3]. Finally, sperm cells, which also use an undulatory swimming gait, move through viscoelastic and shearthinning cervical mucus $[5,7,13,28,33]$. This biomedical relevance, improvements in analytical and numerical techniques, and recent experimental work towards using artificial [9, 17, 23] and biological propulsion [22, 26] for disease detection and drug delivery has driven recent increased interest in modelling swimming at small length scales.

In many systems relevant to medicine, microscale swimmers progress through polymer suspensions such as mucus [15]. Due to the complexity of non-Newtonian fluid modelling, analytical and numerical studies must typically make simplifying assumptions about the underlying system. Examples are two-dimensional (2D) studies extending Taylor's small-amplitude analysis to non-Newtonian rheologies [4, 14, 24, 25, 34], two-dimensional non-Newtonian simulations [19, 20, 31, 32], and three-dimensional (3D) studies with either small-amplitude beating [6] or simplified rheology [7]. Whilst these studies have proven invaluable in shaping our knowledge, intuition, and understanding of microscale swimming, there nevertheless remains a critical role for experimental studies as a means of more closely approximating systems in natura.

Shear-thinning is an important property of polymer suspensions, such as human 
cervical mucus, whereby the suspended polymers align with shear flow allowing them to slip past one another more easily, resulting in regions of lower apparent viscosity. Swimmers in shear-thinning fluids generate a corridor of thinned fluid surrounding themselves that can lead to gains in propulsive velocity $[11,16]$. Recent experimental work has furthermore shown that a low Reynolds number swimmer generates a thinned fluid envelope extending in all directions approximately one body-length in diameter [10]. These experiments also suggested that while shear-thinning rheology increases swimming speed and decreases the cost of swimming relative to a Newtonian fluid of the same zero-shear viscosity, the kinematics and dynamics of an undulatory swimmer in a shear-thinning fluid is nearly identical to that of a Newtonian fluid with the same effective or average viscosity $[8,10]$.

Experimental data acquisition of flow fields driven by microswimmers is typically limited to a two-dimensional slice at swimmer's midplane; the flow shear rate depends upon velocity derivatives, and as such a highly-resolved differentiable flow field is required to probe the effects of shear-thinning rheology. However, while twodimensional data are sufficient to accurately measure the flow field around a planar swimmer, the shear rate and therefore the flow dynamics are dependent upon out-ofplane flow derivatives which must be properly incorporated into the analysis [18]. Ignoring these effects results in relative errors in the shear rate of $25-40 \%$ for Caenorhabditis elegans in a Newtonian fluid [18].

This relative error in shear rate indicates that non-Newtonian effects on both locomotion and the resulting flow field in complex fluids may be larger than anticipated. Examples in which underestimating the local shear rate may yield significant errors include the impact of elastic stretching on locomotion, measured by the Weissenberg number $W i=\lambda_{E} \dot{\gamma}$ where $\lambda_{E}$ is the longest relaxation time of the fluid, and the effects of swimming through a generalized Newtonian fluid, whose shearthinning or shear-thickening behavior is indicated by the Carreau number $\mathrm{Cr}=\lambda_{\mathrm{Cr}} \dot{\gamma}$ where $\lambda_{C r}$ is a timescale that represents the onset of shear-thinning effects [18].

In this study, we experimentally quantify the errors introduced when estimating the cost of swimming in shear-thinning fluids from two-dimensional data without accounting for out-of-plane effects around the low Reynolds number swimmer $C$. elegans. We then derive a scaling argument to show that this error depends approximately linearly upon the power-index of the fluid. We begin with a discussion of the experimental protocols and the equations underlying the flow dynamics.

\section{Methods}

2.1. Experimental Techniques To quantify the impact of out-of-plane factors, we examine the flow fields generated by $C$. elegans using image processing and particle tracking velocimetry techniques. C. elegans, a $1 \mathrm{~mm}$ long nematode (see Fig. 1(a)), swims with a predominately planar sinusoidal swimming gait [29]. We seed shearthinning fluids with tracer particles, which are dilute and do not affect the fluid properties. We then measure the time-periodic flow fields over six to ten beat-cycles 
and use a least-squares fitting algorithm to phase-match each cycle by comparing instantaneous body-shapes. In this way, successive cycles can be folded into one single master cycle, greatly improving our spatial resolution and allowing for the calculation of smooth spatial derivatives of velocity.

In our previous work, we have experimentally explored the differences in the resulting flow fields generated by swimming $C$. elegans as a function of the shearthinning behaviour; we observed ( $i$ ) an increase in the magnitude of vorticity (also predicted theoretically via the waving sheet model by Vélez-Cordero and Lauga [34]), (ii) a redistribution of fluid velocities from head to tail, and (iii) kinematics (e.g. swimming speed $U$ and frequency $f$ ) and mechanical power that scale with a shearthinning fluid's effective viscosity. We note that effective viscosity is defined as the average viscosity experienced by a nematode using the characteristic shear rates of its swimming gait $0.35 \lesssim \dot{\gamma} \lesssim 15 \mathrm{~s}^{-1}[8,10]$.

In this study, we focus on measurements of the cost of swimming, or mechanical power, expended by $C$. elegans in shear-thinning fluids. We experimentally obtain three components to compute the mechanical power of $C$. elegans: (i) the instantaneous position of the fluid-worm interface $S$ via image processing, (ii) a spatially differentiable flow field $\boldsymbol{u}$ from particle tracking techniques, and (iii) a constitutive model for the fluid stresses $\sigma$ from rheology and the Carreau-Yasuda model (see Section 2.2). To estimate $S$, we multiply the observed body contours by the diameter of the nematode's body $(80 \mu \mathrm{m})$ to form a thin surface area. Figure 1(c,d) show the streamlines at a particular phase of the nematode beating cycle generated experimentally from particle tracking velocimetry in a Newtonian and a representative shear-thinning fluid, respectively. We note that the body shapes are approximate. For more details on the techniques and data see $[8,10,18]$.

2.2. Fluids \& Rheology Following previous studies [8, 10], we consider the swimming of $C$. elegans through sufficiently viscous fluids so that the ratio of viscous to inertial forces, the Reynolds number, is small: $R e \lesssim 0.1$. In such viscous flows, the dynamics of the fluid may be modelled via the inertialess generalized Stokes flow equations

$$
\boldsymbol{\nabla} \cdot \boldsymbol{\sigma}=\mathbf{0}, \quad \boldsymbol{\nabla} \cdot \boldsymbol{u}=0,
$$

where $\boldsymbol{u}$ is the fluid velocity and $\boldsymbol{\sigma}$ is the stress tensor

$$
\sigma=-p \boldsymbol{I}+\eta(\dot{\gamma}) \dot{\gamma}
$$

with $\dot{\gamma}=|\dot{\gamma}| \equiv \sqrt{\frac{1}{2}(\dot{\gamma}: \dot{\gamma})}$ the magnitude of the shear rate tensor $\dot{\gamma} \equiv\left(\nabla \boldsymbol{u}+\nabla \boldsymbol{u}^{\top}\right)$ and $p$ the pressure. For Newtonian fluids, the fluid viscosity $\eta$ is constant; however for rate-dependent fluids, the viscosity $\eta(\dot{\gamma})$ depends upon this flow shear rate $\dot{\gamma}$. Thus, the equations governing shear-thinning flow are nonlinear, making 3D analytical and numerical approaches difficult.

We prepare shear-thinning fluids by adding small amounts of the polymer xanthan gum $\left(\mathrm{XG}, 2.7 \times 10^{6} \mathrm{MW}\right.$, Sigma Aldrich G1253) to water in the presence of salt. 
The XG concentration in buffer ranges from $50 \mathrm{ppm}$ to $3000 \mathrm{ppm}$, and solutions are well-mixed. These aqueous XG solutions have been well-characterized and have negligible elasticity [8, 10, 27]. We characterize all fluids (Newtonian and shearthinning) using a cone-and-plate rheometer (strain-controlled RFS III, TA Instruments) at a range of constant shear rates. At the lowest concentration $\left(c_{\mathrm{XG}}=50 \mathrm{ppm}\right)$, the behaviour of the XG solutions is approximately Newtonian, while we find strong shear-thinning behaviour (e.g. power-law viscosity) for the most concentrated XG solutions (Fig. 1(b)); we note that we cannot independently tune shear-thinning behaviour and bulk viscosity since both quantities increase with additional polymer. We quantify this behaviour by fitting the rheological measurements with the CarreauYasuda model [2]:

$$
\eta(\dot{\gamma})=\eta_{\infty}+\left(\eta_{0}-\eta_{\infty}\right)\left(1+\left(\lambda_{C r} \dot{\gamma}\right)^{2}\right)^{\frac{n-1}{2}},
$$

where $\eta_{0}$ is the zero-shear viscosity, $\eta_{\infty}$ is the infinite-shear viscosity, and $n$ is the power-law index. The Carreau time scale $\lambda_{C r}$ is the inverse of the shear rate at which shear-thinning effects become significant.

\section{Results and discussion}

3.1. Correcting for out-of-plane contributions Given an experimental planar flow field $u(x, y), v(x, y)$, we want to calculate the shear rate field to obtain a viscosity field. In component form the total shear magnitude is given by

$$
\dot{\gamma}_{3 \mathrm{D}}=\left[2 u_{x}^{2}+\left(u_{y}+v_{x}\right)^{2}+2 v_{y}^{2}+2 w_{z}^{2}+\left(u_{z}+w_{x}\right)^{2}+\left(v_{z}+w_{y}\right)^{2}\right]^{1 / 2},
$$

which contains non-trivial components in the $z$-direction that are not captured by experiment. Under the condition that worm kinematics are planar, $v_{z}=u_{z}=0$ by symmetry. Furthermore, by incompressibility, $w_{z}=-u_{x}-v_{y}$. Using these conditions, which are valid for a range of non-Newtonian flows, the 3D formula for shear rate in the midplane can be written in terms of the available 2D data [18],

$$
\dot{\gamma}_{3 \mathrm{D}}=\dot{\gamma}_{\mathrm{pl}}=\left[2 u_{x}^{2}+2 v_{y}^{2}+\left(u_{y}+v_{x}\right)^{2}+2\left(u_{x}+v_{y}\right)^{2}\right]^{1 / 2} .
$$

We can then examine the effect of neglecting these out of plane contributions by calculating the fluid viscosity $\eta_{3 D}$ based upon the full $3 \mathrm{D}$ shear rate $\dot{\gamma}_{3 \mathrm{D}}$, and the viscosity $\eta_{2 D}$ based upon the $2 \mathrm{D}$ shear rate $\dot{\gamma}_{2 \mathrm{D}}$,

$$
\dot{\gamma}_{2 \mathrm{D}}=\left[2 u_{x}^{2}+\left(u_{y}+v_{x}\right)^{2}+2 v_{y}^{2}\right]^{1 / 2} .
$$

Since the 3D shear rate magnitude must be greater than or equal to the $2 \mathrm{D}$ shear rate magnitude $\left(\dot{\gamma}_{3 \mathrm{D}} \geq \dot{\gamma}_{2 \mathrm{D}}\right)$, the uncorrected planar (2D) data systematically overestimates local viscosities $\left(\eta_{3 \mathrm{D}} \leq \eta_{2 \mathrm{D}}\right)$ and therefore also overestimates the cost of swimming $\left(P_{3 \mathrm{D}} \leq P_{2 \mathrm{D}}\right)$ in a shear-thinning fluid via the Carreau-Yasuda model (3). Figure 2 shows the overestimate in viscosity $\left(\eta_{2 D}-\eta_{3 D}\right) / \eta_{3 D} \geq 0$ for a variety of fluids at a particular beat phase. 


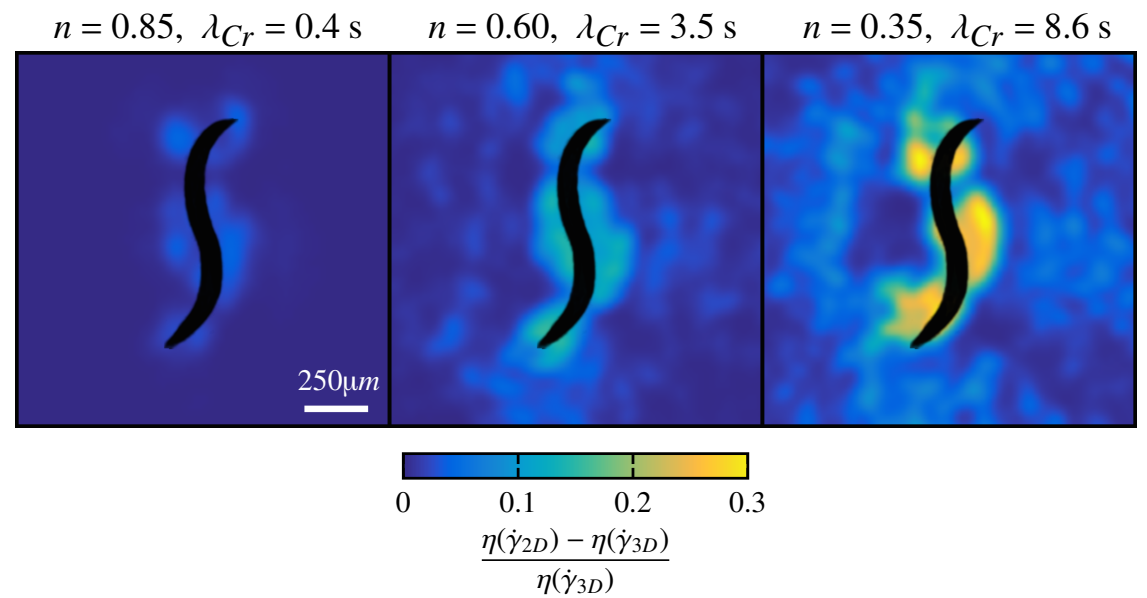

Figure 2. (Colour available online) Error in computing local viscosity via the Carrea-Yasuda model (Eq. 3) using the "planar" shear rate (Eq. 5) and the experimentally-measured 2D shear rate (Eq. 6) for three different shear-thinning fluids, from nearly Newtonian (left) to highly shear-thinning (right).

3.2. Calculating the cost of swimming With knowledge of the velocity, shear rate, and viscosity fields, the cost of swimming or mechanical power is a simple accounting of the rate of energy expenditure required to deform a swimmer's body in a viscous medium. Here, we estimate the cost of swimming by integrating the viscous and pressure forces at the swimmer-fluid interface assuming a no-slip condition.

For a translating body in Stokes flow, the differential force $\mathrm{d} \boldsymbol{F}$ on a given element of the swimmer's surface $\mathrm{d} S$ is

$$
\mathrm{d} \boldsymbol{F}=\boldsymbol{n} \cdot \boldsymbol{\sigma} \mathrm{d} S
$$

where $\boldsymbol{n}$ is the outward normal vector from the swimmer's surface. Since a microorganism is freely swimming with no external forces or torques acting upon its body, it is instantaneously force-free and the integral of $\boldsymbol{F}$ over the surface $S$ is zero. Note that the local forces are only dependent on body shape of the swimmer and fluid stresses from the aforementioned fundamental equations (1).

With knowledge of the local forces, we then calculate the local mechanical power (or rate of work)

$$
\mathrm{d} P=-\mathrm{d} \boldsymbol{F} \cdot \boldsymbol{u}=-\boldsymbol{n} \cdot \boldsymbol{\sigma} \cdot \boldsymbol{u} \mathrm{d} S,
$$

where $\boldsymbol{u}$ is the velocity of the surface of the swimmer. Integrating over the full surface of the swimmer, we obtain the cost of swimming

$$
P=-\int_{S} \boldsymbol{n} \cdot \boldsymbol{\sigma} \cdot \boldsymbol{u} \mathrm{d} S .
$$

We then incorporate the flow fields and body geometries obtained via image processing and particle techniques to find the typical cost of swimming over a full beating cycle 


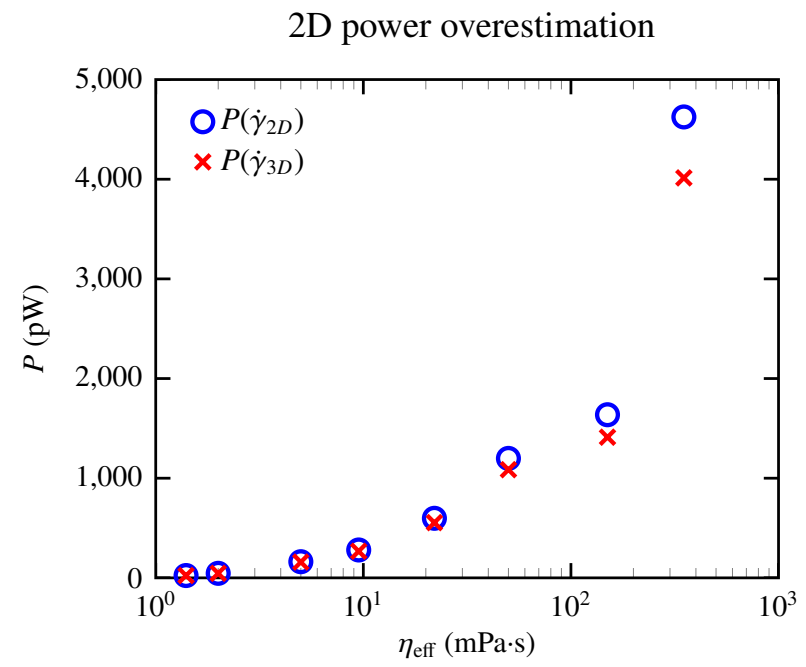

FIGURE 3. (Colour available online) Cost of swimming (mechanical power, Eq. 9) calculated via the experimentally-measured 2D shear rate (open circles, (6)) and the corrected "planar" shear rate (solid plusses, (5)). Data represent calculations in different fluids with varying rheological parameters with $1>n>0.3$ and are shown as a function of average or "effective" viscosity $\eta_{\text {eff }}$; increased effective viscosity corresponds to increased polymer concentration and thus shear-thinning behaviour. Note that with increasing shear-thinning, the difference between the estimated power grows.

(see [10] for more detail). We perform this calculation twice using the same data. First, we compute $P_{3 D}$ using the 3D shear rate (5) and second $P_{2 D}$ using the 2D shear rate (6); A summary of these data are shown in Figure 3 as a function of the average or effective viscosity $\eta_{\text {eff }}$ experienced by the swimmer; increasing effective viscosity indicates increasing polymer concentration and shear-thinning behaviour. Since the shear rate magnitude is greater when out of plane derivatives are included, the viscosity of a shear-thinning fluid in 3D is lower than would be expected from 2D calculations; we therefore anticipate our 3D estimate of power to be lower than that calculated without the out-of-plane correction. Indeed, we find $\eta_{3 D} \leq \eta_{2 D}$ and therefore $P_{3 D} \leq P_{2 D}$; furthermore, the discrepancy between $P_{3 D}$ and $P_{2 D}$ grows with increasing shear-thinning behaviour.

3.3. Quantifying the out-of-plane error With the cost of swimming in shearthinning fluids calculated using both shear rate formulae, we can now determine the error introduced by considering a 2D flow field without applying an out-ofplane correction. Since we have planar beating, in the midplane $\mathbf{n}=\left[n_{x}, n_{y}, 0\right]$ and $\mathbf{u}=[u, v, 0]$ so that out-of-plane components in $\sigma$ do not play a part when the power is estimated via the midplane velocity field. The only difference appears in the calculation of $\eta(\dot{\gamma})$ using the 2D (6) versus 3D (5) shear rate formulae.

Figure 3 details the difference between power calculated with the 2D (6) and 3D (5) formulae, demonstrating that indeed the true cost of swimming is lower than 
previously calculated using the $2 \mathrm{D}$ estimate of shear rate. How significant is this error, and can its importance be easily predicted a priori for a given system? Since we have

$$
P \propto \mathbf{n} \cdot \boldsymbol{\sigma} \cdot \mathbf{u},
$$

we observe that

$$
\begin{aligned}
& P_{2 D} \propto \mathbf{n} \cdot\left(-p \boldsymbol{I}+\tau_{2 D}\right) \cdot \mathbf{u}=\mathbf{n} \cdot\left(-p \boldsymbol{I}+\eta_{2 D} \dot{\gamma}_{2 D}\right) \cdot \mathbf{u}, \\
& P_{3 D} \propto \mathbf{n} \cdot\left(-p \boldsymbol{I}+\tau_{3 D}\right) \cdot \mathbf{u}=\mathbf{n} \cdot\left(-p \boldsymbol{I}+\eta_{3 D} \dot{\gamma}_{2 D}\right) \cdot \mathbf{u},
\end{aligned}
$$

since in the midplane $\mathbf{n} \cdot \dot{\gamma}_{3 D} \cdot \mathbf{u}=\mathbf{n} \cdot \dot{\gamma}_{2 D} \cdot \mathbf{u}$, which can be seen more clearly in component form,

$$
\begin{aligned}
& \left(\begin{array}{lll}
n_{1}, & n_{2}, & 0
\end{array}\right) \cdot\left(\begin{array}{lll}
\dot{\gamma}_{11} & \dot{\gamma}_{12} & \dot{\gamma}_{13} \\
\dot{\gamma}_{21} & \dot{\gamma}_{22} & \dot{\gamma}_{23} \\
\dot{\gamma}_{31} & \dot{\gamma}_{32} & \dot{\gamma}_{33}
\end{array}\right) \cdot\left(\begin{array}{c}
u_{1} \\
u_{2} \\
0
\end{array}\right)=n_{1}\left(\dot{\gamma}_{11} u_{1}+\dot{\gamma}_{12} u_{2}\right)+n_{2}\left(\dot{\gamma}_{21} u_{1}+\dot{\gamma}_{22} u_{2}\right) \\
& =\left(n_{1}, \quad n_{2}\right) \cdot\left(\begin{array}{ll}
\dot{\gamma}_{11} & \dot{\gamma}_{12} \\
\dot{\gamma}_{21} & \dot{\gamma}_{22}
\end{array}\right) \cdot\left(\begin{array}{l}
u_{1} \\
u_{2}
\end{array}\right) .
\end{aligned}
$$

When we evaluate the surface integral for power, this midplane line is projected to the whole surface as a best approximation of what is possible with planar data. Note also that by incompressibility, components of the form $u_{x}+v_{y}$ are included in the 3D viscosity calculation via $w_{z}=-\left(u_{x}+v_{y}\right)$. Examining the relative error in the power used by the worm, we then see

$$
\frac{P_{3 D}-P_{2 D}}{P_{3 D}} \approx \frac{\mathbf{n} \cdot\left(\eta_{3 D} \dot{\gamma}_{2 D}-\eta_{2 D} \dot{\gamma}_{2 D}\right) \cdot \mathbf{u}}{\mathbf{n} \cdot \eta_{3 D} \dot{\gamma}_{2 D} \cdot \mathbf{u}}=\frac{\eta_{3 D}-\eta_{2 D}}{\eta_{3 D}} .
$$

For ease of notation, we write $\dot{\gamma}_{3 D}-\dot{\gamma}_{2 D}=k$, for some variable $k$ that may depend upon the degree of shear-thinning, and write $\alpha=n-1$. Then,

$$
\begin{aligned}
& \eta_{2 D}=\eta_{\infty}+\left(\eta_{0}-\eta_{\infty}\right)\left(1+\lambda^{2} \dot{\gamma}_{2 D}^{2}\right)^{\alpha / 2}, \\
& \eta_{3 D}=\eta_{\infty}+\left(\eta_{0}-\eta_{\infty}\right)\left(1+\lambda^{2}\left(\dot{\gamma}_{2 D}+k\right)^{2}\right)^{\alpha / 2} .
\end{aligned}
$$

Assuming that for most shear-thinning fluids, $\eta_{0} \gg \eta_{\infty}$ [34], and for worm swimming $\lambda \dot{\gamma} \gg 1$ [18], we have the ratio

$$
\frac{\eta_{2 D}}{\eta_{3 D}} \approx \frac{\eta_{0}\left(\lambda^{\alpha} \dot{\gamma}_{2 D}^{\alpha}\right)}{\eta_{0}\left(\lambda^{\alpha}\left(\dot{\gamma}_{2 D}+k\right)^{\alpha}\right)}=\frac{\dot{\gamma}_{2 D}^{\alpha}}{\left(\dot{\gamma}_{2 D}+k\right)^{\alpha}}=\left[1+\frac{k}{\dot{\gamma}_{2 D}}\right]^{-\alpha} .
$$

Since $0 \leq k / \dot{\gamma}_{2 D}<1$, we thus have the first-order expansion,

$$
\frac{\eta_{2 D}}{\eta_{3 D}} \approx 1-\frac{|k|}{\dot{\gamma}_{2 D}} \alpha
$$

so that

$$
\frac{P_{3 D}-P_{2 D}}{P_{3 D}}=\frac{\eta_{3 D}-\eta_{2 D}}{\eta_{3 D}} \approx 1-\left[1-\frac{k}{\dot{\gamma}_{2 D}} \alpha\right],
$$


Scaling law comparison

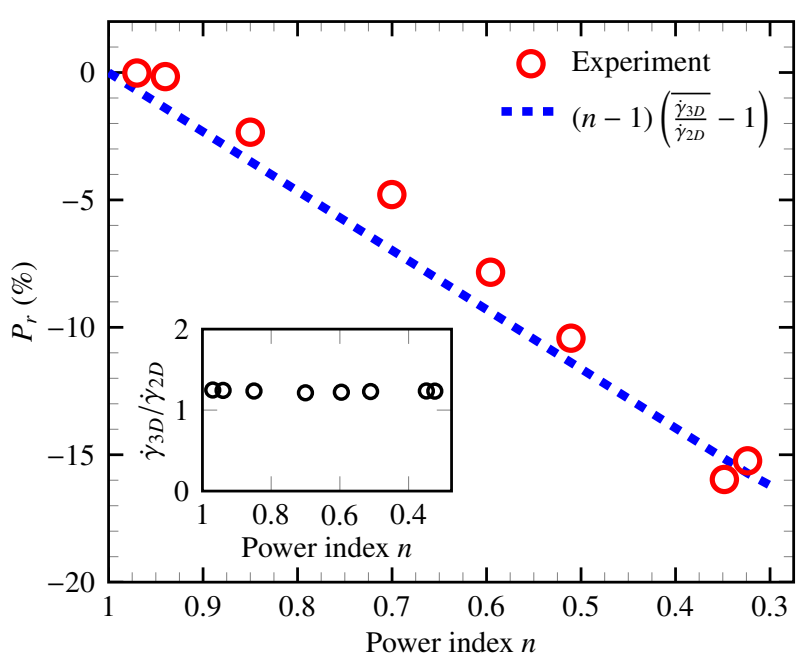

Figure 4. (Colour available online) Power ratio $P_{r}=\left(P_{3 D}-P_{2 D}\right) / P_{3 D}$ versus shear thinning index $n$, calculated with a separate $\dot{\gamma}_{3 D} / \dot{\gamma}_{2 D}$ for each fluid. The linear scaling (Eq. 20, dashed line) is given for constant average $\dot{\gamma}_{3 D} / \dot{\gamma}_{2 D}=1.23$. The ratio $\dot{\gamma}_{3 D} / \dot{\gamma}_{2 D}$ is inset as a function of $n$ and demonstrates little variation across all power indices.

and finally, representing $\frac{P_{3 D}-P_{2 D}}{P_{3 D}}$ as the power ratio $P_{r}$, we see that

$$
P_{r} \approx(n-1) \frac{\dot{\gamma}_{3 D}-\dot{\gamma}_{2 D}}{\dot{\gamma}_{2 D}}=(n-1)\left(\frac{\dot{\gamma}_{3 D}}{\dot{\gamma}_{2 D}}-1\right)
$$

giving

$$
P_{r} \approx(n-1)\left(\frac{\dot{\gamma}_{3 D}}{\dot{\gamma}_{2 D}}-1\right),
$$

which for the Newtonian case $(n=1)$ is zero, as expected.

For only slightly changing worm kinematics [8], we expect that the shear rate does not vary appreciably with the degree of shear-thinning [16]; furthermore, experiments have suggested that kinematics in shear-thinning and Newtonian fluids of the same effective viscosity are nearly identical and fairly insensitive to changes in bulk viscosity. The effective viscosity of a fluid is defined as the average viscosity experienced by the worm over its range of characteristic shear rates [10]. Because these experimentally-measured kinematics seem to be largely independent of shearthinning effects, this implies that the swimmer is imposing similar boundary conditions and therefore similar fluid velocities and shear rates despite different degrees of shear thinning behaviour $[16,10]$. We therefore hypothesize that the ratio $\dot{\gamma}_{3 D} / \dot{\gamma}_{2 D}$ may be approximately constant across all experiments. 
To test this hypothesis, we return to our experimental data and calculate the average shear rate measured in our flow field over a full beating cycle; to reduce noise, we only consider shear rates equal to or greater than $2 \%$ of the maximum shear rate measured during the cycle. Indeed, we find that the ratio of the typical time averaged 3D to 2D shear rates $\overline{\dot{\gamma}_{3 D} / \dot{\gamma}_{2 D}}$ has a mean of 1.23 and a standard deviation of just 0.01 (Fig. 4, inset), and thus we expect a linear dependence in the relative error in the power calculation as a function of the power-law index $n$. Substituting $\overline{\dot{\gamma}_{3 D} / \dot{\gamma}_{2 D}}$ into equation (19), we have

$$
P_{r} \approx(n-1)\left(\frac{\dot{\gamma}_{3 D}}{\dot{\gamma}_{2 D}}-1\right) .
$$

We now compare this linear scaling to our measured power ratio $P_{r}$ as a function of power-law index $n$. With no parameter fitting, we see that this linear scaling shows good agreement with experimental data (Fig. 4). These results suggest that the inaccuracies introduced by ignoring out-of-plane effects for an undulatory swimming gait in a generalized Newtonian fluid can be quantified simply with rheological properties and an estimate of the fluid shear rate normal to the beating plane, which can be easily obtained via the incompressibility condition and available $2 \mathrm{D}$ shear rate data.

\section{Conclusion}

Microscale swimming via planar undulations through suspensions of polymers is of direct importance to a number of medically-relevant systems, such as human reproduction and disease. Whilst the nonlinear nature of such fluids can make analytical and numerical study difficult, experimental studies provide an effective means of closely approximating these systems in natura, as well as improving and validating modelling. Experimental flow field data can be used to probe important swimmer biophysical quantities, for instance power expenditure. Such quantities depend on flow derivatives, and so highly-resolved flow-fields are required for accurate estimation, restricting results to two-dimensional data in the swimmer midplane. However, out-of-plane derivatives can be accounted for via symmetry arguments [18], and here we show that neglecting to include these components results in an overestimate of the power expended by a nematode worm in a shear-thinning fluid. Under certain approximations [10], this overestimate was shown to depend on the calculation of the effective viscosity in the swimmer midplane, and for the data considered could be as high as $16 \%$. By applying a simple scaling argument, we show that this overestimate varies approximately linearly with the power-law index $n$ of the shear-thinning fluid, reaching good agreement with experimental data. As imaging techniques improve, it will become feasible to reconstruct differentiable flow fields around smaller-scale swimmers, such as human sperm and spirochetes, which exhibit planar beating to propel through non-Newtonian fluids. It will then be important to include out-of-plane contributions in the analysis of such studies. 


\section{Acknowledgments}

D. A. Gagnon and T. D. Montenegro-Johnson contributed equally to this work. We thank P. E. Arratia and E. Lauga for helpful discussions. This work was supported by NSF-CBET-PMP-1437482. T. D. M.-J. was supported by a Royal Commission for the Exhibition of 1851 Research Fellowship.

\section{References}

[1] “Microbiology by numbers", Nat. Rev. Micro 9 (2011) 628-628; 10.1038/nrmicro2644.

[2] P.J. Carreau, D.C.R. DeKee, R.P. Chhabra, "Rheology of polymeric systems", Hanser: Munich (1997).

[3] N. W. Charon, A. Cockburn, C. Li, J. Liu, K. A. Miller, M. R. Miller, M. A. Motaleb, and C. W. Wolgemuth, "The unique paradigm of spirochete motility and chemotaxis", Ann. Rev. Microbiol. 66 (2012) 349-370; 10.1146/annurev-micro-092611-150145.

[4] G. Cupples, R. J. Dyson, and D. J. Smith, "Viscous propulsion in active transversely isotropic media", J. Fluid Mech. 812 (2017) 501-524; 10.1017/jfm.2016.821.

[5] L.J. Fauci and R. Dillon, "Biofluidmechanics of Reproduction", Annu. Rev. Fluid Mech. 38 (2006) 371-394; 10.1146/annurev.fluid.37.061903.175725.

[6] H. C. Fu, C. W. Wolgemuth, and T. R. Powers, "Swimming speeds of filaments in nonlinearly viscoelastic fluids", Phys. Fluids 21 (2009) 033102-033110; 10.1063/1.3086320.

[7] G. R. Fulford, D. F. Katz, and R. L. Powell, "Swimming of spermatozoa in a linear viscoelastic fluid", Biorheology 35 (1998) 295-309; 10.1016/S0006-355X(99)80012-2.

[8] D. A. Gagnon, N. C. Keim, and P. E. Arratia, "Undulatory swimming in shear-thinning fluids: experiments with Caenorhabditis elegans", J. Fluid Mech. 758 (2014) R3; 10.1017/jfm.2014.539.

[9] D. A. Gagnon, N. C. Keim, X.-N. Shen, and P. E. Arratia "Fluid-induced propulsion of rigid particles in wormlike micellar solutions", Phys. Fluids 26 (2014) 103101; 10.1063/1.4896598.

[10] D. A. Gagnon, and P. E. Arratia, "The cost of swimming in generalized Newtonian fluids: experiments with C. elegans", J. Fluid Mech. 800 (2016) 753-765; 10.1017/jfm.2016.420.

[11] S. Gómez, F. A. Godínez, E. Lauga, and R. Zenit, "Helical propulsion in shear-thinning fluids", Journal of Fluid Mechanics 812 (2017) R3; 10.1017/jfm.2016.807.

[12] K. L. Hill, "Parasites in motion: flagellum-driven cell motility in African trypanosomes", Current Opinion in Microbiology 13 (2010) 459-465; 10.1016/j.mib.2010.05.015.

[13] D. F. Katz and S. A. Berger, "Flagellar propulsion of human sperm in cervical mucus", Biorheology 17 (1980) 169-175; 10.3233/BIR-1980-171-218.

[14] M. S. Krieger, S. E. Spagnolie, and T. Powers, "Microscale locomotion in a nematic liquid crystal", Soft Matter 11 (2015) 9115-9125; 10.1039/c5sm02194d.

[15] S. K. Lai, Y.-Y. Wang, D. Wirtz, J. Hanes, "Micro-and macrorheology of mucus", Adv. Drug Deliv. Rev. 61 (2009) 86-100, 10.1016/j.addr.2008.09.012.

[16] G. Li and A. M Ardekani, "Undulatory swimming in non-Newtonian fluids", J. Fluid Mech. 784 (2015) R4; 10.1017/jfm.2015.595.

[17] R. Mhanna, F. Qiu, L. Zhang, Y. Ding, K. Sugihara, M. Zenobi-Wong, and B.J. Nelson, "Artificial Bacterial Flagella for Remote-Controlled Targeted Single-Cell Drug Delivery", Small 10 (2014) 1953-1957; 10.1002/smll.201303538.

[18] T. D. Montenegro-Johnson, D. A. Gagnon, E. Lauga, and P. E. Arratia, "Flow analysis of the low Reynolds number swimmer C. elegans", Phys. Rev. Fluids 1 (2016) 053202; 10.1103/PhysRevFluids.1.053202.

[19] T. D. Montenegro-Johnson, A. A. Smith, D. J. Smith, D. Loghin, and J. R. Blake, "Modelling the fluid mechanics of cilia and flagella in reproduction and development", Eur. Phys. J. E 35 (2012) $111 ; 10.1140 /$ epje/i2012-12111-1.

[20] T. D. Montenegro-Johnson, D. J. Smith, and D. Loghin, "Physics of rheologically enhanced 
propulsion: Different strokes in generalized Stokes", Phys. Fluids 25 (2013) 081903; 10.1063/1.4818640.

[21] K. M. Ottemann and A. C. Lowenthal, "Helicobacter pylori uses motility for initial colonization and to attain robust infection", Infection and immunity 70 (2002) 1984-1990; 10.1128/IAI.70.4.1984-1990.2002.

[22] D. Park, S. J. Park, S. Cho, Y. Lee, Y. K. Lee, J.-J. Min, B. J. Park, S. Y. Ko, J.-O. Park, and S. Park, "Motility Analysis of Bacteria-Based Microrobot (Bacteriobot) Using Chemical Gradient Microchamber", Biotechnol. Bioeng. 111 (2013) 134-143; 10.1002/bit.25007.

[23] T. Qiu, T. -C. Lee, A. G. Mark, K. I. Morozov, R. Munster, O. Mierka, S. Turek, A. M. Leshansky, and P. Fischer, "Swimming by reciprocal motion at low Reynolds number", Nat. Commun. 5 (2014) 5119; 10.1038/ncomms6119.

[24] E. E. Riley and E. Lauga, "Enhanced active swimming in viscoelastic fluids", Euro. Phys. Lett. 108 (2014) 34003; 10.1209/0295-5075/108/34003.

[25] E. E. Riley and E. Lauga, "Small-amplitude swimmers can self-propel faster in viscoelastic fluids", J. Theoret. Biol. 382 (2015) 345-355; 10.1016/j.jtbi.2015.06.045.

[26] M. S. Sakar, E. B. Steager, D. H. Kim, A. A. Julius, M.-J. Kim, V. Kumar, and G. J. Pappas, "Modeling, control and experimental characterization of microbiorobots", Int. J. Rob. Res. 30 (2011) 647-658; 10.1177/0278364910394227.

[27] X. N. Shen and P. E. Arratia, "Undulatory Swimming in Viscoelastic Fluids", Phys. Rev. Lett. 106 (2011) 208101; 10.1103/PhysRevLett.106.208101.

[28] S. S. Suarez, "Mammalian Sperm Interactions with the Female Reproductive Tract", Cell Tissue Res. 363 (2016) 185-194; 10.1007/s00441-015-2244-2.

[29] J. Sznitman, X.-N. Shen, R. Sznitman, and P. E. Arratia, "Propulsive force measurements and flow behavior of undulatory swimmers at low Reynolds number", Phys. Fluids 22 (2010) 121901; $10.1063 / 1.3529236$.

[30] G. I. Taylor "Analysis of the Swimming of Microscopic Organisms", Proc. R. Soc. Lon. Ser-A 209 (1951) 447-461; 10.1098/rspa.1951.0218.

[31] J. Teran, L. Fauci, and M. Shelley "Viscoelastic Fluid Response Can Increase the Speed and Efficiency of a Free Swimmer", Phys. Rev. Lett. 104 (2010) 038101; 10.1103/PhysRevLett.104.038101.

[32] B. Thomases and R. D. Guy "Mechanisms of Elastic Enhancement and Hindrance for FiniteLength Undulatory Swimmers in Viscoelastic Fluids", Phys. Rev. Lett. 113 (2014) 098102; 10.1103/PhysRevLett.113.098102.

[33] C. -K. Tung, C. Lin, B. Harvey, A.G. Fiore, F. Ardon, M. Wu, and S.S. Suarez, "Fluid viscoelasticity promotes collective swimming of sperm", Sci. Rep. 7 (2017) 3152; 10.1038/s41598-017-03341-4.

[34] J. N. Vélez-Cordero and E. Lauga, "Waving transport and propulsion in a generalized Newtonian fluid”, J. Non-Newton. Fluid. 199 (2013) 37-50; 10.1016/j.jnnfm.2013.05.006. 